“ C 2019 IEEE. Personal use of this material is permitted. Permission from IEEE must be obtained for all other uses, in any current or future media, including

reprinting/republishing this material for advertising or promotional purposes, creating new collective works, for resale or redistribution to servers or lists, or reuse of any copyrighted component of this work in other works." 


\title{
DETECTION OF MICROPLASTICS USING MACHINE LEARNING
}

\author{
ZENON CHACZKO ${ }^{1}$, PETER WAJS-CHACZKO ${ }^{2}$, DAVID TIEN ${ }^{3}$, YOUSEF HAIDAR ${ }^{1}$ \\ ${ }^{1}$ University of Technology Sydney, Ultimo, 2007, Australia \\ ${ }^{2}$ Macquarie University, Macquarie Park, 2109, Australia \\ ${ }^{3}$ School of Computing \& Mathematics, Charles Sturt University, NSW, Australia \\ E-MAIL:Zenon.Chaczko@uts.edu.au,Peter.Wajs-Chaczko@students.mq.edu.au,dtien@csu.edu.au, \\ Yousef.Haidar@uts.edu.au
}

\begin{abstract}
:
Monitoring the presence of micro-plastics in human and animal habitats is fast becoming an important research theme due to a need to preserve healthy ecosystems. Microplastics pollute the environment and can represent a serious threat for biological organisms including the human body, as they can be inadvertently consumed through the food chain. To perceive and understand the level of microplastics pollution threats in the environment there is a need to design and develop reliable methodologies and tools that can detect and classify the different types of the microplastics. This paper presents results of our work related to exploration of methods and techniques useful for detecting suspicious objects in their respective ecosystem captured in hyperspectral images and then classifying these objects with the use of Neural Networks technique.
\end{abstract}

\section{Keywords:}

Microplastics; Machine learning; Neural network; Identification; Classification; Hyperspectral image

\section{Introduction}

Microplastics are fast becoming persistent contaminants in both aquatic and terrestrial environments [1-4]. There is an increasing scholarly work about the presence and negative effects of microplastics on marine environments [2, 3]. So far, a relatively small number of reports can be found about the need for studying contamination sources, pollution characteristics and ecological impact of microplastics. There is mounting evidence that macroplastics can influence soil biota at different trophic levels [4], and thus pose a serious threat to animal and human health in the food web. Further in-depth investigation is required to uncover the full impact and define ecological threats related to microplastics at various levels and in all kinds of biological ecosystems. To study the impact of microplastics on various environments and humans, researchers require better analytical methodologies and tools which would allow to determine the characteristics of pollution and the impact of macroplastics on the aquatic, soils and aerial ecology. However, there is an absence of standardised protocols, methodologies, best practices and tools for the quantification [4]. And there is an urgent need for the uniformity of methods, procedures and protocols related to microplastics presence, extraction and their classification (identification). This research aims to address this need. In order to better understand the level and impact of microplastics pollution, methods, computational solutions and sensing devices that can detect and classify the different types of the microplastics urgently required $[4,5]$. In the field of plastic recycling there are some useful and practical tools present; hyperspectral imaging is on of most promising technologies, for it successes [5-9].

The main objectives and outcomes of this research work project is to explore a reliable approach for finding an effective computing solution for locating objects of interest in an image (canvas), as well as, to perform classification of these objects for correct identification.

\section{A. Scope, Aims \& Objectives}

At this stage, this research is only concerned about exploring methods of detecting the number of objects along with their respective location in a hyperspectral image, whilst the classification of objects applies the Neural Networks. Thus, the main objectives and outcomes of this project is to find a reliable method for:

- locating objects in an image/canvas

- classifying objects

The proposed Neural Network-based solution is to employ the supervised learning approach which needs data to be used as training samples. Even though there are other methods of training Neural Network models without data, such as the unsupervised learning approach, they still require 
some information about the application or scenario; in the unsupervised learning approach a way of observing a model or a system is required to adjust and learn, to reach the optimal solution. In this project's scenario it is unrealistic to have a model that simulate the way plastics react to light synthetically and accurately to the real world. Thus, the supervised learning approach was chosen.

\section{Methodology}

As previously stated, a dataset is required for training the Neural Network model, but none were to be found in the public domain. Some creative thinking was needed to keep the research alive and one main idea stood out; that idea was to use a dataset of hyperspectral images of objects, and discarding the requirement of having them be images of microplastics. The motivation was, that at this stage this research project is concerned about seeing if hyperspectral images can be used effectively in Neural Networks, to apply that in the field of microplastics pollution research.

The "SpecTex" (University of Eastern Finland n.d.) dataset was used for this project [9]. This dataset includes spectral images of sixty textile samples with different texture patterns [9]. In this research case, those samples can be considered as (suspicious contaminant) objects. The images have a size of $640 \times 640 \times 39$, as in image high times image width times number of channels. The number of channels refer to different snapshot of the object in different wavelengths, ranging from $400-\mathrm{nm}$ to $780-\mathrm{nm}$ with a $10-\mathrm{nm}$ increment. To prepare the data for training, a limited number of files (10), that contain the samples, were selected for the experiment (see TABLE 1).

TABLE 1. The list of files selected for the experiment

$\begin{array}{ll}\text { T01.tif } & \text { T02.tif } \\ \text { T03.tif } & \text { T04.tif } \\ \text { T05.tif } & \text { T06.tif } \\ \text { T10.tif } & \text { T11.tif } \\ \text { T14.tif } & \text { T60.tif }\end{array}$

A random point was taken from each file, and using that point $28 \times 28 \times 39$ sized images were cropped at the center of that point to simulate plastics that were broken down to small pieces by the sun and ocean waves. Then the cropped images were slightly distorted by using a circular mask to simulate the shape of a pellet. This process was repeated 100 times each file, and another 10 times for each files. The first time to generate the training samples and the second time to generate the evaluation/testing samples. However, there were two regions that were chosen for each type of data samples to be generated from (see Fig. 1). The smaller region is used for the testing as it fewer samples to be generated are needed than the training samples. The reason behind this separation is because to further ensure that the evaluation samples are new data that are not seen in the training phase; this will give an indication of how the system will perform on unseen data.

\section{A. Tools And Libraries}

The following selection of software development environment and analytical tools were explored, integrated and used in the experimental solution:

- Python - main language used

- tensorflow - GPU enabled library for building Neural Networks

- $\quad$ numpy - data manipulation module (library)

- matplotlib - graphing and plotting

- tensorboard - graphing and plotting

- scikit-learn - used for the confusion matrix and for shuffling data

- scikit-image - used for finding the contours for object detection

- panda - usually used for data manipulation, but in this case, it was opportunistically used for displaying tables

- Jupyter - a playground for quickly and conveniently experimenting with python

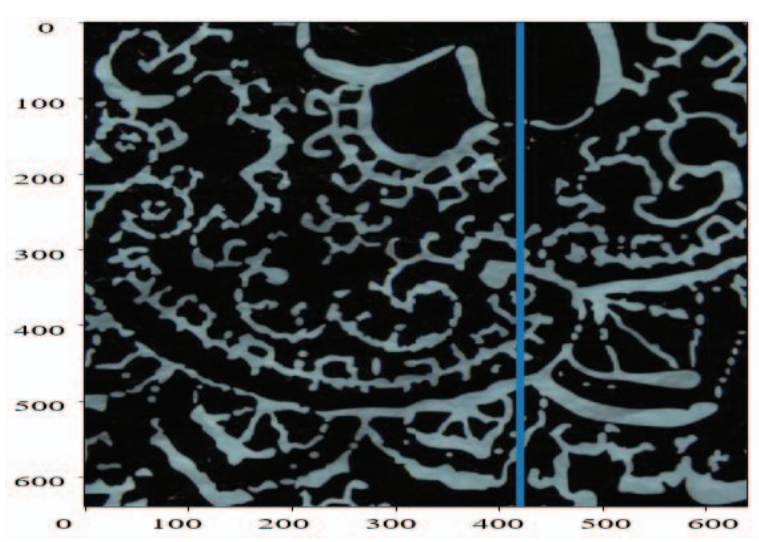

FIGURE 1. A single layer of 39 layers in the T14.tif file

\section{Key Concepts}

In 2013, Zhang et al. in their research work [8] on tensor discriminative locality alignment and spectral-spatial feature extraction in hyperspectral image discussed dimensionality reduction of hyperspectral data using the Principal Component Analysis (PCA) approach. The purpose was to remove the redundancy of information and thus reduce the size of the data. The redundancy comes from the inter-band correlation which is can be very high, and it can be reduced, with a rather low chance of losing significant information. However, giving the fact that the 
size of the data set used in this initial phase was rather small to begin with, PCA was not considered but not employed in the project. Also, Zhang et al [8] introduced a very useful concept of tensor representation of hyperspectral images (HSIs) that we used to put forward the HSI feature extraction, which is called tensor discriminative locality alignment (TDLA) method. In our experimentation, the tensor representation of HSIs concept was employed. The HSIs are composed of several images/layers, that represent a spectralchannel/spectral- wavelength. Images/layers could be then represented by a grayscale image, where the values of pixel represent the intensity of the light wave in that pixel corresponding to the spectral-channel.

\section{The design and implementation of Machine Learning Solution}

The Machine Learning system need to execute in the deployment state. It is assumed that when the system is deployed, its computing model is already trained. The deployed system (see Fig. 2) executes the following steps:

1. The objects/microplastics are placed in a canvas

2. A hyperspectral image is taken of the whole canvas

3. One layer of the hyperspectral image is supplied to the Object Detector

4. The locations of the objects are supplied to the "crop\&Resize" module

5. All the layers are supplied to the "crop\&Resize" module

6. Cropped and correctly resized hyperspectral images is supplied to the Neural Network model

7. The Neural Network provides the prediction

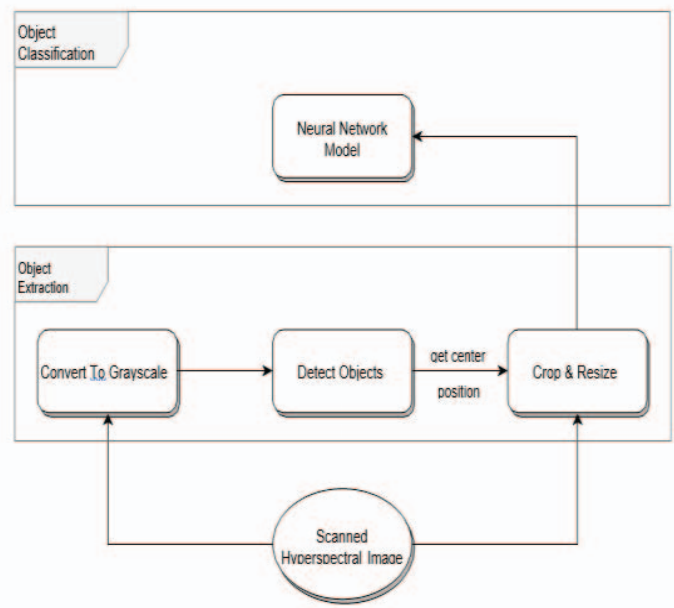

FIGURE 2. System Design - Already Trained NN Model
Note, Step 5 can also be placed before Step 3, since the cropping and resizing can only happen after all the first 5 steps are completed. However, the rest of the steps should be in their respective order.

\section{A. Object Detection}

Assume that the figure $3 \mathrm{a}$ below as an example of a grayscale image of an object, applying the find contours function from scikit-image, will yield what is shown in figure $3 \mathrm{~b}$. The con- tour's maximum and minimum values in both the $\mathrm{Y}$ and $\mathrm{X}$ axis, are calculated to construct the bounding-box of the object, and then using that boundingbox the image is then cropped as shown in 3c. That cropped image is then resized to fit the input specification of the Neural Network, but that is not shown here. It is important to note that the cropped image will not be the grayscale image but rather all the 39 layers of different wavelength data, but this is shown just for clarity of what would happen.

The goal of this process is to automate the extraction of multiple objects within a single snapshot, to properly feed them to the Neural Network model. For example, the Figure 4a below shows a $500 \times 500$ canvas where objects are randomly placed in it, and the noise was introduced to see the reliability of the method; the Figure $4 \mathrm{~b}$ show the result of the find contours function, which detected 6 objects and colored them differently. Similarly, the max and min is used to extract the objects (Fig. 4c). Again, the actual extraction/cropping will be applied to all 39 layers for each object (Fig. 5); In the Figure 5 it is hard to distinguish the difference between each layer; remember that each layer represents an image of a wavelength, ranging from 400- $\mathrm{nm}$ to $780-\mathrm{nm}$ with a $10-\mathrm{nm}$ increment. However, a computer algorithm or for this case a trained Neural Network model, could possibly recognise the subtle differences and use them to correctly classify the objects.
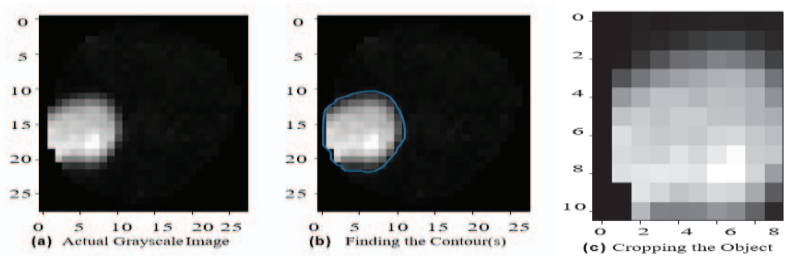

FIGURE 3. Object detection example 

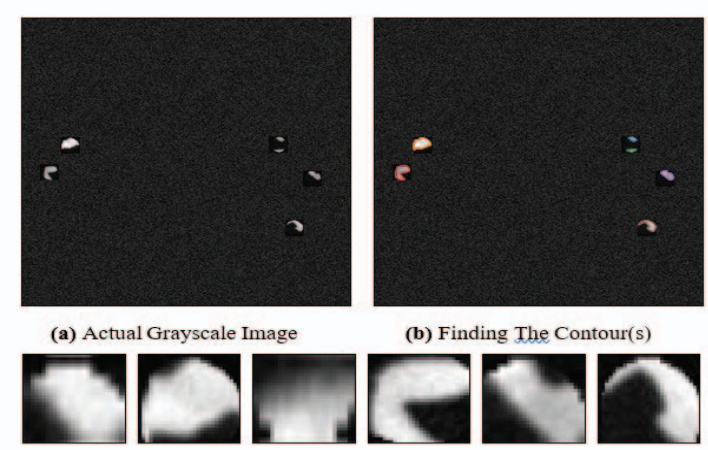

(c) Extracting The Objects

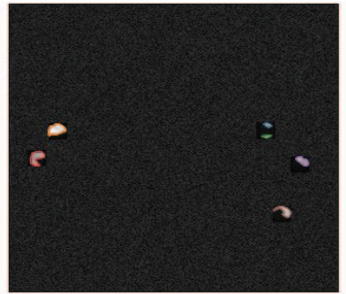

(b) Finding The Contour(s)

extraction

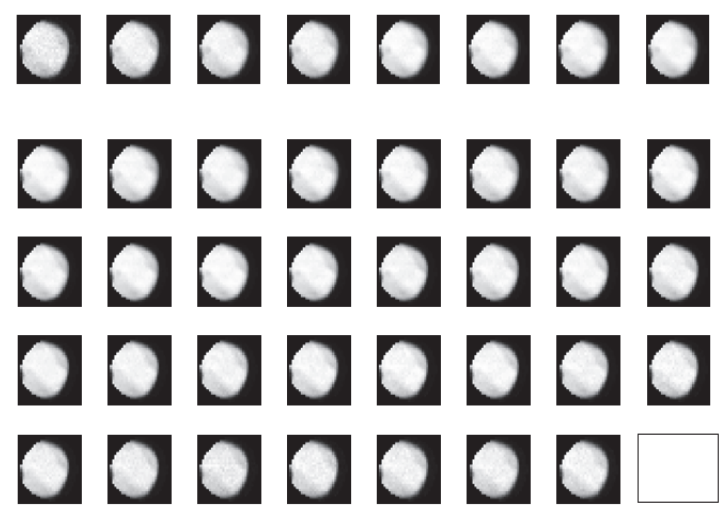

FIGURE 5. Example of all 39 Layers of an object

The contours of every object are being calculated and used to crop the objects as shown in LISTING 1. The loop in line 5 , is a secondary filter that ensure that the found contour must have more than 30 points representing it, because otherwise, the found contour is likely representing a anomaly or noise in the image rather than an actual object. Line 13 and 14 show how the bounding box are calculated from the points in the contour.

LISTING 1. Object Detection and Extraction

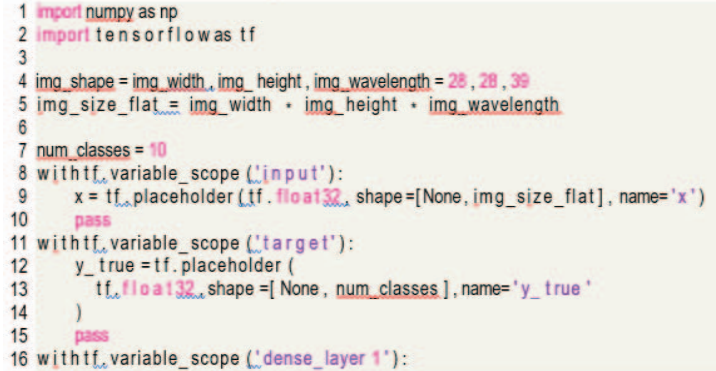

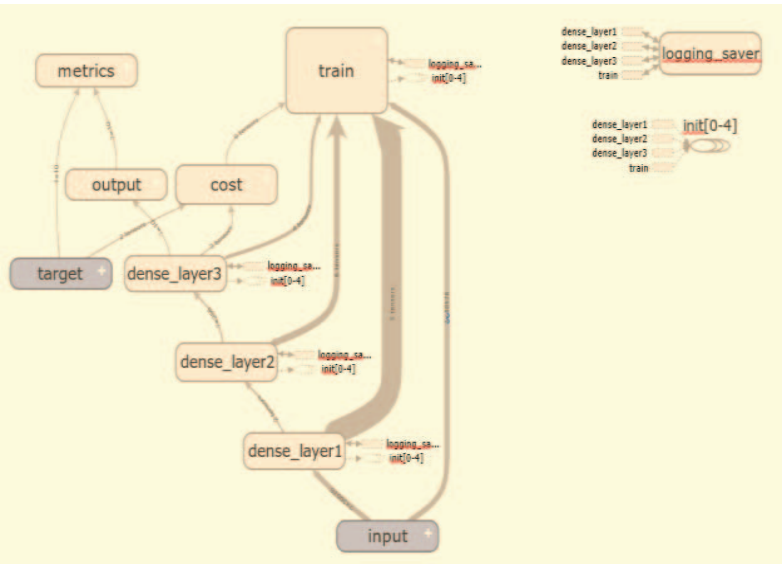

FIGURE 6. Neural Network Implementation Design

\section{B. Object Classification}

This part of the system was implemented using tensorflow; this section will describe the implementation details, along with tensorflow's layers and train application programing interface(API). The structure of the system is as follow, the input layer, three fully-connected dense layers, and lastly the output layer; each layer, along with the training component shall be discussed in the sections below. Also, there will be an extra section that describes the other, less important, components. The layers API is a python module that contains a set of functions to construct Neural Network related layers, such as convolution layer, denser layer, and more. The way it is designed allow for stacking of layers, as in the output of the previous layer can be passed to the next layer. Neural Network programing especial the backpropagation stage is highly error prone; the layers API take care of the low-level implementation details, and leaves high-level design up to the user.

Moreover, tensorflow's train API will take care of the backpropagation process. By using both modules, this will hence eliminate most of the chances of introducing errors in the model. The Figure 6 below shows the graph generated by the tensorboard tool; while the tool only allows exporting the graph in "png" format, one can merely copy the $s v g$ code from the Internet browser and convert it to a pdf file for a better quality, which is what was done and shown in the figure.

All layers, except the 3rd dense layer, have a rectified linear unit or a RLU as their activation function. The RLU can be defined with the equation below.

$$
f(x)=x+=\max (0, x)
$$


Also the weights and biases were initialized with the xavier initializer provided by tensorflow; it is consider to be the state of the art technique for initializing the weights. Rather than initializing the weights randomly using this function is assumed to have less chance for the Neural Network to get stuck on the local minimum of the cost function in the training stage, and hence, it get a better chance of finding the global minimum.

LISTING 2. Neural Network Model's Source Code

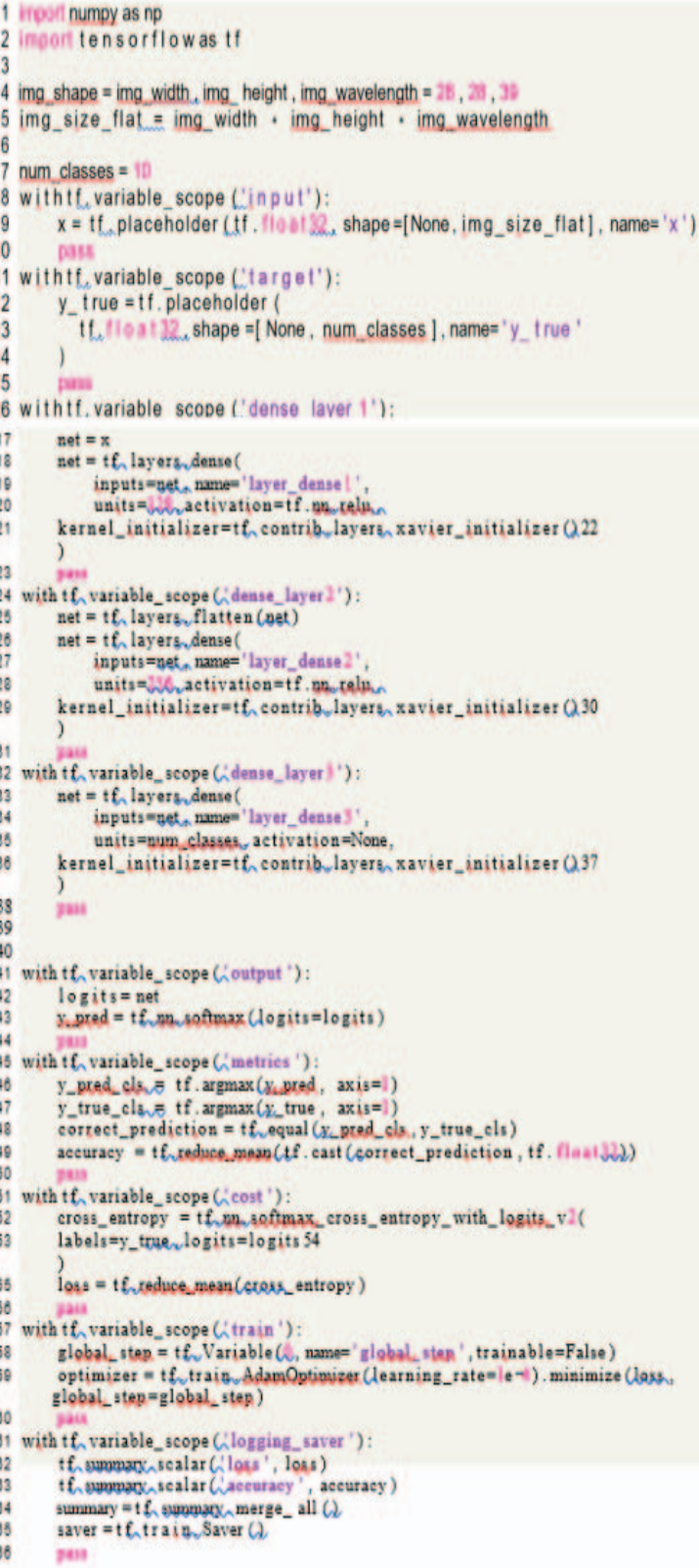

\section{Input Layer}

The "target" component, shown in Figure 6 is an input, that contain the true label; it is only used in training, and not in prediction mode.

TABLE 2. Input Data Shape

\begin{tabular}{lll} 
& Data Shape Description & Data Shape $^{1}$ \\
\hline X_image & $\begin{array}{l}\text { batch size } \\
\text { flattened image size: } 28 \times 28 \times 39 \\
\text { image width } \times \text { image height } \times \text { No. } \\
\text { channels }\end{array}$ & 20 \\
& 30576 \\
y_true & batch size & \\
& No. Classes & 20 \\
'for example x image shape is then 20 by 30576. & 10 \\
\hline
\end{tabular}

${ }^{2}$ The size 20 was chosen arbitrarily.

D. Densely Connected Layers

TABLE 3. Densely Connected Layers Data Shape

\begin{tabular}{lll} 
& Data Shape Description & Data Shape \\
\hline dense_layer1 & $\begin{array}{l}\text { batch size } \\
\text { No. dense_layer1's neurons }\end{array}$ & 20 \\
& & 128 \\
dense_layer2 & $\begin{array}{l}\text { batch size } \\
\text { No. dense_layer2's neurons }\end{array}$ & 20 \\
& 256 \\
dense_layer3 & $\begin{array}{l}\text { batch size } \\
\text { No. dense_layer3's neurons which } \\
\text { has to be JN- classes }\end{array}$ & 20 \\
& 10 \\
\hline
\end{tabular}

E. Output Layer

TABLE 4. Output Layer Data Shape

\begin{tabular}{lll} 
& Data Shape Description & Data Shape \\
\hline prediction_softmax & batch size & 20 \\
& No. Classes & 10 \\
\hline
\end{tabular}

\section{F. Training Components}

The "softmax_cross_entropy_with_logits" function supplied by tensorflow's API is used to represent the cost functions. The cross entropy function require the actual true labels in order to calculate the cost. The calculated cost is then reduced by averaging the container tensor on the batch axis, in other words the shape of the tensor would be [1 10$]$ rather than the original [20 10] shape. The averaging help in smoothing the learning curve. This is what is called batch mode; no conclusive research on literature was done by this report, but batch mode was used in most examples on the Internet, so batch mode was automatically chosen over stochastic mode. After that the cost is supplied to the training component, which is just an "AdamOptimizer", that is implemented by tensorflow. The tensorflow API provide 
different optimizers, and all of them managed the backpropagation process; it incorporates the weights regulation process, which requires the gradient that is calculated from the loss; the gradient calculation is also managed by the optimizer. The optimizer requires a scalar value to define the learning rate, so the value $110-4$ was picked. The "AdamOptimizer" was picked as it seemed to be the state of the art algorithm that every article/tutorial used.

\section{G. Related Components}

The "metrics" and the "logging_saver" are the only components left unmentioned. Both components are related and they are unnecessary for the application, but are used for gathering metrics about the training phase progress. Logs are captured on every 10th iteration of training steps, and they contain the accuracy-and-loss, resulted within each training step/batch. The data is shown in Figure 7.

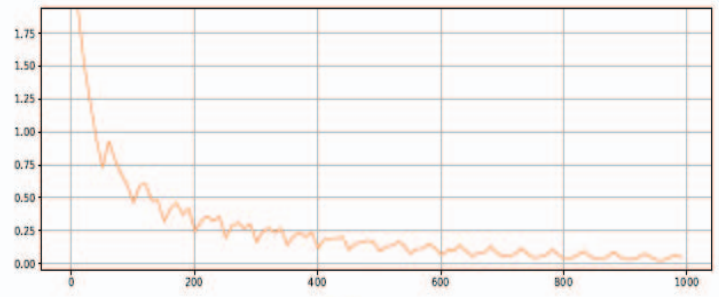

(a) Loss

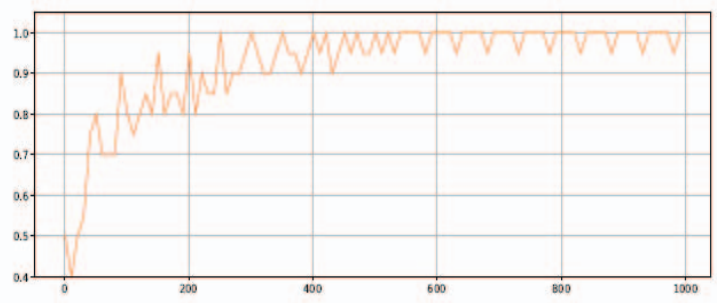

(b) Accuracy

FIGURE 7. Metrics Over Training

\section{Experimental Results}

This section will only focus on the object classification part, or the Neural Network model; that is because the object detection is a well-known area and the results taken from the experiments were to be expected, and are already shown in previous sections. There were two stages in the building of the Neural Network model, to be discussed; the optimization stage and the evaluation stage. Both sections will include a brief description of how these results were realized and gathered.

\section{A. Optimization}

The sample data used for training had a size of 1000 . The data were shuffled in a random order using function designed for this purpose from the scikit-learn tool; the function merely shuffles the data and there is nothing special about it, but it was used, nonetheless, merely for convenience. As discussed earlier, the results are generated (Fig. 7), using the data collected from the "logging_saver" component, which logs scalar values every 10 iteration of training steps; the data were conveniently accessible from the tensorboard tool. The loss results (Fig. 7) show that these values continue to decrease until they stabilise. The loss value refers to how much of a change is needed to minimize the error and reach the optimal solution. Just because the loss has stabilized, it does not necessarily mean that it had reached the optimal solution; in fact, there is no global method, across all scenarios and situations, that proves that the found solution is optimal. To do that one would need specific knowledge on the field of interest. On the other hand, the accuracy value (Fig. 7b) refers to the accuracy over the training batch, and it does not necessarily evaluate or prove that the model is perfect, like how it is shown to be reaching a $100 \%$ accuracy. However, it does show that, at least, within the training dataset, the model is figuring out the relationship between different features in the data and classifying them correctly.

LISTING 3. Optimization Process - Source Code

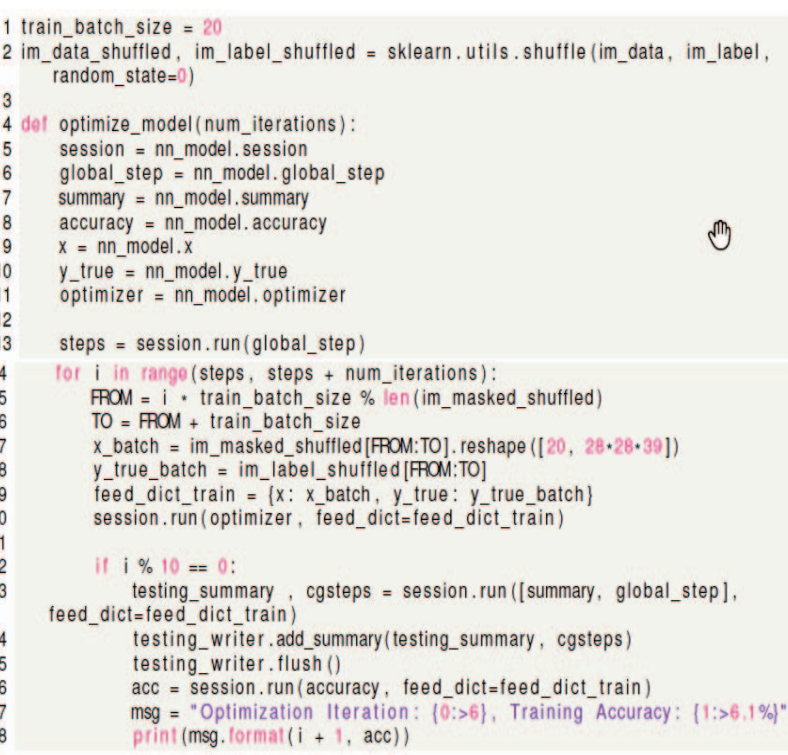


LISTING 4. The Optimization Process Output

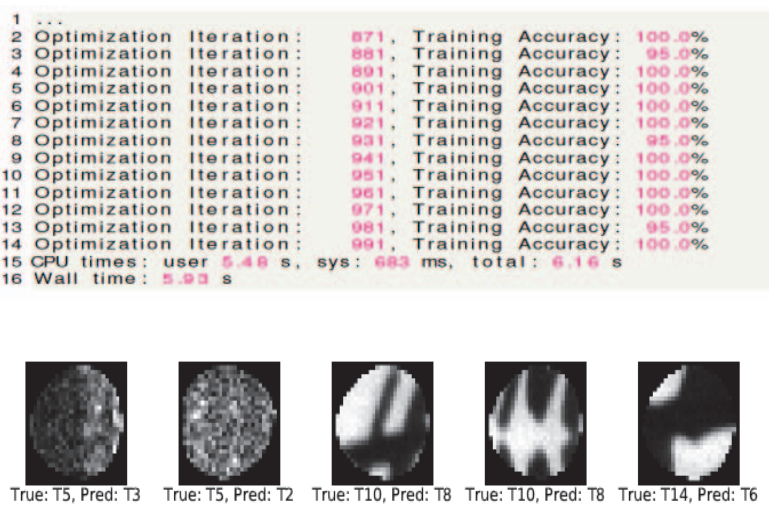

FIGURE 8. Falsely Classified Images - After Optimization

\section{B. Evaluation of Results}

After a thousand iteration of training, the evaluation resulted in an accuracy of $95 \%$, with a 95 correct classifications out of 100 samples, that were never seen by the Neural Network model. The figure 8 below show all 5 misclassified objects. While this may seem impressive, it is not an indication of performing as good in the real-world scenario. That is because it is unclear what did the Neural Network model learn from the training phase; for example, the features that it learned about and used, does not exist in hyperspectral images of microplastics. The question is then, can the Neural Network, given a proper dataset, find features that distinguish the types of different microplastics, during the training phase. As previously state, hyperspectral imaging of plastics is used in the classification of plastics in the recycling industry, so it is highly likely that the answer to that question is yes. However, given the fact that no data about hyperspectral imaging of microplastics were available in public, at the time of this report was made, it is impossible to have a comprehensive conclusion, a one that is free of assumptions. In the end, these results do indicate the possibility of Neural Networks model, trained with hyperspectral images of microplastics, being applicable.

TABLE 5. Confusion Matrix - After Optimization

\begin{tabular}{|c|c|c|c|c|c|c|c|c|c|c|c|}
\hline \multicolumn{12}{|c|}{ Predicted as } \\
\hline & & o & 1 & 2 & 3 & 4 & 5 & 6 & 7 & 8 & 9 \\
\hline & 0 & 10 & 0 & o & o & o & o & o & o & o & o \\
\hline & 1 & o & 10 & 0 & o & o & 0 & 0 & 0 & 0 & 0 \\
\hline & 2 & o & o & 10 & 0 & o & o & o & o & o & o \\
\hline & 3 & o & 0 & 0 & 10 & o & 0 & o & o & 0 & 0 \\
\hline & 4 & o & 0 & 1 & 1 & 8 & o & o & o & o & o \\
\hline & 5 & o & o & o & o & o & 10 & o & o & o & o \\
\hline & 6 & o & 0 & o & o & o & 0 & 8 & 0 & 2 & o \\
\hline & 7 & 0 & o & o & o & 0 & 0 & 0 & 10 & o & o \\
\hline & 8 & o & o & 0 & 0 & o & 0 & 1 & 0 & 9 & 0 \\
\hline & 9 & 0 & o & o & 0 & 0 & o & o & o & o & 10 \\
\hline
\end{tabular}

LISTING 5. Evaluation Process - Source Code

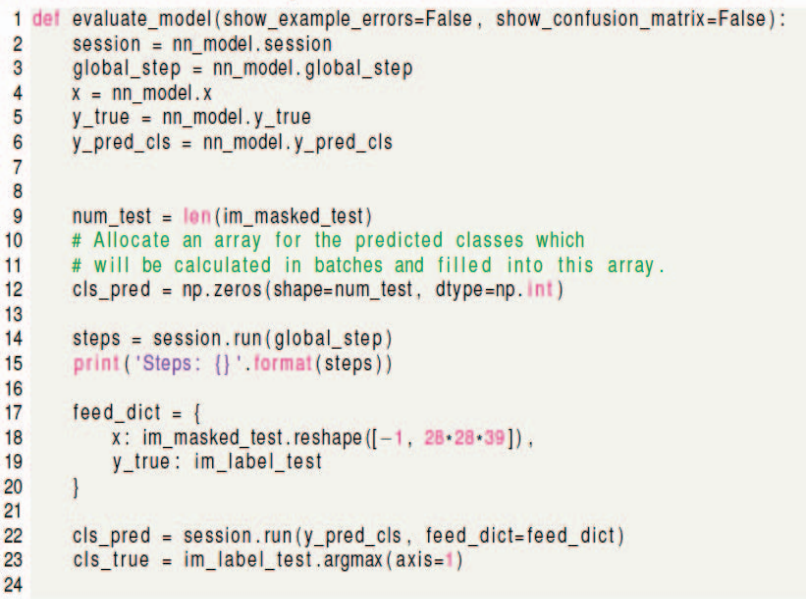

\section{Conclusions}

This paper has demonstrated how the Machine Learningbased solutions can be designed and built for the effective identification and classification of microplastics objects. A viable, practical and possibly standardised solution for these tasks can be archived by incorporating and integrating various public domain advanced tools and libraries. However, it was found, if we cannot solely rely on such a solution alone. Each time, when somehow a newer set of data is introduced, it is likely some further changes and adaptations will be still required. This is because opensource tools in most cases address very generic solutions to the algorithm and rarely these solutions cater for the adaption and their continued improvement to satisfy the required accuracy.

\section{References}

[1] Browne, M.A., Galloway, T. and Thompson, R., 2007. Microplastic - an emerging contaminant of potential concern? Integrated environmental assessment and Management, 3(4), pp.559-561.

[2] Lusher, A., 2015. Microplastics in the marine environment: distribution, interactions and effects. In Marine anthropogenic litter (pp. 245-307). Springer, Cham.

[3] Hidalgo-Ruz, V., Gutow, L., Thompson, R.C. and Thiel, M., 2012. Microplastics in the marine environment: a review of the methods used for identification and quantification. Environmental science \& technology, 46(6), pp.3060-3075.

[4] Defu He, Yongming Luo, Shibo Lua, Mengting Liu, Yang Song Lili Leia, 2018. Microplastics In Soils: 
Analytical Methods, Pollution Characteristics And Ecological Risks TrAC Trends in Analytical Chemistry, Volume 109, Dec 2018, pp. 163-172.

[5] Chaczko Z., Kale A., Santana-Rodríguez José Juan, Suárez-Araujo Carmen Paz, 2018. Towards an IOT Based System for Detection and Monitoring of Microplastics in Aquatic Environments, IEEE 22nd International Conference on Intelligent Engineering Systems (INES 2018), June 21 2018, Las Palmas, Spain, pp. 000057-000062

[6] Kale A., Chaczko, Z., Evolutionary Feature Optimization and Classification for Monitoring Floating Objects, Computational Intelligence and Efficiency in Engineering Systems, Volume 595 of the series Studies in Computational Intelligence, Springer 2015, pp.3-16.

[7] Karaca, A.C., Ertürk, A., Güllü, M.K., Elmas, M. \& Ertürk, S. 2013, 'Plastic waste sorting using infrared hyperspectral imaging system', pp. 1-4.

[8] Zhang, L., Zhang, L., Tao, D. \& Huang, X. 2013, 'Tensor Discriminative Locality Alignment for Hyperspectral Image Spectral-Spatial Feature Extraction', IEEE Transactions on Geo-science and Remote Sen,

[9] University of Eastern Finland n.d., Spectex | UEF, University Website, Finland, viewed 28 May 2018, $<$ https://www.uef.fi/web/spectral/spectex>., 2015) (pp. 107-112). 